\title{
Highly photoconductive amorphous carbon nitride films prepared by cyclic nitrogen radical sputtering
}

\author{
T. Katsuno and S. Nitta \\ Department of Electrical Engineering, Gifu University, Yanaido 1-1, Gifu 501-1193, Japan \\ H. Habuchi \\ Department of Electrical and Computer Engineering, Gifu National College of Technology, Shinsei-Chou, \\ Motosu, Gifu 501-0495, Japan \\ V. Stolojan and S. R. P. Silva ${ }^{\text {a) }}$ \\ Advanced Technology Institute, School of Electronics and Physical Sciences, University of Surrey, Guildford \\ Surrey GU2 7XH, United Kingdom
}

(Received 29 December 2003; accepted 23 July 2004)

\begin{abstract}
We report on the growth of amorphous carbon nitride films $\left(\mathrm{a}-\mathrm{CN}_{x}\right)$ showing the highest conductivity to date. The films were prepared using a layer-by-layer method (a-CN $\left.\mathrm{N}_{x}: \mathrm{LL}\right)$, by the cyclical nitrogen radical sputtering of a graphite radical, alternated with a brief hydrogen etch. The photosensitivity $S$ of these films is $10^{5}$, defined as the ratio of the photoconductivity $\sigma_{p}$ to the dark conductivity $\sigma_{d}$ and is the highest value reported thus far. We believe that the carriers generated by the monochromatic light (photon energy $6.2 \mathrm{eV}$ ) in the a- $\mathrm{CN}_{x}$ : $\mathrm{LL}$ films are primarily electrons, with the photoconductivity shown to increase with substrate deposition temperature. (0) 2004 American Institute of Physics. [DOI: 10.1063/1.1792384]
\end{abstract}

The family of tetrahedral amorphous carbon films has shown promise toward device applications based on luminescence. In particular, hydrogenated tetrahedral amorphous carbon has shown relatively good photoconducting properties ${ }^{1-3}$ which can be further improved by the addition of impurities such as nitrogen. ${ }^{1,4-6}$ The resulting amorphous carbon nitride films $\left(\mathrm{a}-\mathrm{C}: \mathrm{N}_{x}\right)$ show interesting properties, including electroluminescence, ${ }^{7}$ photoluminescence, ${ }^{8}$ low dielectric constant ${ }^{9}$ and detection capability for heavy-ion particle. ${ }^{10}$ The layer-by-layer amorphous carbon nitride films $\left(\mathrm{a}-\mathrm{CN}_{x}: \mathrm{LL}\right)$ prepared in this study show a two to three orders of magnitude improvement in photoconductivity reported for any form of a-C films. We investigated the density $\rho, s p^{3}$ fraction and nitrogen concentration [obtained using an electron-energy-loss spectroscopy (EELS)], the optical energy gap $E_{04}$, the Urbach energy $E_{U}$ [calculated from photothermal deflection spectroscopy (PDS)], and defect density [from electron spin resonance (ESR)] as a function of substrate temperature $T_{S}$ during sputtering, in order to examine the thermal evolution of the growth and structure affecting the photoconductivity of these films.

The a-CN $\mathrm{CN}_{x}$ :LL films were prepared by a cyclic process of a deposition step of thin a- $\mathrm{CN}_{x}$ film by a nitrogen radical sputtering, immediately followed by a hydrogen treatment step that helps to reconstruct the bulk material; this process is repeated until the required thickness of film is grown. The hydrogen treatment on the a-CN $\mathrm{CN}_{x}$ films helps to reduce the defect density and remove weak $\mathrm{C}$ dangling bonds without the inclusion of significant atomic hydrogen. ${ }^{4}$ The a- $\mathrm{CN}_{x}$ films were prepared using sputtering in a $13.56 \mathrm{MHz}$ rf system, at a power of $85 \mathrm{~W}$, a substrate temperature $T_{S}$ that varies from room temperature (RT) to $400^{\circ} \mathrm{C}$, and a $\mathrm{N}_{2}$ sputter gas pressure of 0.12 Torr (gas purity of $99.999 \%$ ). For the

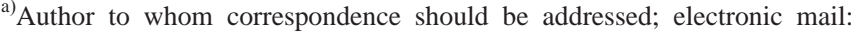
s.silva@surrey.ac.uk hydrogen treatment step, we used a glow discharge of $\mathrm{H}_{2}$ at 0.50 Torr (gas purity $99.999 \%$ ) at a rf power of $85 \mathrm{~W}$ at $T_{S}$. The a- $\mathrm{CN}_{x}$ films initially grown are reduced with structural reconstruction during this phase. Typical film thicknesses per layer for a single-sputter growth and etching cycle are estimated to be 15 and $5 \mathrm{~nm}$, respectively, for a growth time of 3 min, followed by $\mathrm{H}_{2}$ etching for $40 \mathrm{~s}$. We ran seven cycles, resulting in a film thickness of $70 \mathrm{~nm}$. Deposition and etching rates for individual layers are estimated to be in the order of 3.0 and $7.5 \mathrm{~nm} / \mathrm{min}$, respectively. The heating and cooling rates during the film preparation were maintained at 4 and $1{ }^{\circ} \mathrm{C} / \mathrm{min}$, respectively.

The photoconductivity was measured using aluminum (Al) gap electrodes, comprised of structures with a lateral gap of $60 \mu \mathrm{m}$, in 6-mm-wide stripes. A light source from a Xe lamp of $150 \mathrm{~W}$ was used to irradiate the gap cell with a monochromatic light. The photon flux, $F\left(\mathrm{~cm}^{2} \mathrm{~s}\right)^{-1}$, was calculated using a voltage lock-in-amplifier configuration (Stanford research system SR830), with a pyroelectric detector (Hamamatsu Photonics K.K.P2613) at a chopping frequency of $3.5 \mathrm{~Hz}$. The photocurrent passing through the a-CN $\mathrm{CN}_{x}$ : LL film was monitored using a Keithley 6512 picoammeter. The dependence of the photoconductivity on photon energy, at a fixed photon number and applied voltage of $32 \mathrm{~V} \mathrm{dc}$, is shown in Fig. 1. The photon number $F$ used for the experiments for the low-energy range of $3.5-4.6 \mathrm{eV}(5$ $\times 10^{13} \mathrm{~cm}^{-2} \mathrm{~s}^{-1}$ ) was different from the one used for the high energy at $4.5-6.2 \mathrm{eV}\left(1 \times 10^{12} \mathrm{~cm}^{-2} \mathrm{~s}^{-1}\right)$. The photoconductivity of the a-CN $\mathrm{CN}_{x}$ :LL films increased with substrate temperature $T_{S}$ and photon energy, whereas the dark conductivity $\sigma_{d}$, measured at room temperature was $10^{-14}(\Omega \mathrm{cm})^{-1}$ or lower. The photosensitivity, $S=\sigma_{p} / \sigma_{d}$, is approximately $10^{5}$ at a photon energy of $6.2 \mathrm{eV}$, with an energy flux of $5 \mu \mathrm{W} / \mathrm{cm}^{2}$. As the mobility gap of the a-C films has been shown to be larger than that of the optical band gap, ${ }^{2,6}$ which in turn is smaller than the energy used to measure photocon- 


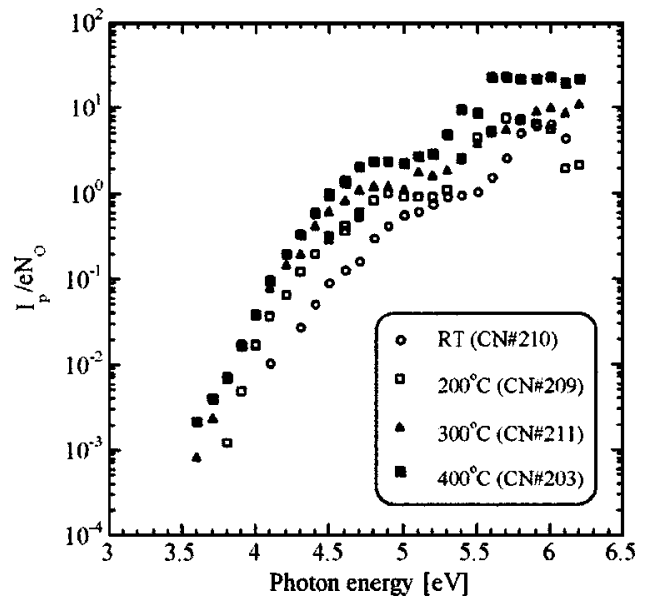

FIG. 1. Dependence of $I_{p} / e N_{0}$ on the photon energy for a-CN $\mathrm{N}_{x}: \mathrm{LL}$, as a function of substrate temperature $T_{s}$, for a fixed photons number. $I_{p}, e$, and $N_{0}$ are the photocurrent $[A]$, the electronic charge $[C]$ and photon number $(\mathrm{s})^{-1}$, respectively. The photon number $N_{0}$ is equal to the product of the photon flux $F\left(\mathrm{~cm}^{2} \mathrm{~s}\right)^{-1}$ with the area of a-CN ${ }_{x}$ :LL film $(6 \mathrm{~mm} \times 60 \mu \mathrm{m})$ irradiated by the monochromatic light.

ductivity $(6.2 \mathrm{eV})$, it follows that the observed photoconductivity is considerably higher than those measured prior to this study.

$S p^{2}$-hybridized carbon has $\pi-\pi^{*}$ and $\sigma-\sigma^{*}$ bondingantibonding bands, with all bonding states filled. In the band model used here, due to the high concentration of nitrogen in the films, a nitrogen lone pair state is believed to form in the $\pi$ valence band. ${ }^{11,12}$ The increase of signal in the photoconductivity at $3.5 \mathrm{eV}$ in Fig. 1 can be attributed to a transition from a nitrogen lone pair state in the valence band to a $\sigma^{*}$ empty antibonding state in the conduction band $\left(E_{\mathrm{NLP}-\sigma^{*}}\right){ }^{10}$ Due to the high optical penetration depth, it follows that the optical absorption is not sufficient in the films examined in this study (film thickness of $70 \mathrm{~nm}$ ) to show photoconductivity below incident photon energies of $3.5 \mathrm{eV}$.

Figure 2 shows the dependence of the defect density $N_{S}$ and nitrogen concentration for a-CN $\mathrm{CN}_{x}$ : LL films as a function of substrate temperature $T_{S}$. Previous reports have shown that the photoconductivity of the a-C films is related to the defect density $N_{S}$, varying in the range of $0.5 \sim 1$ $\times 10^{18} \mathrm{~cm}^{-3}$, and decreasing very slightly with an increasing $T_{S}$. Furthermore, the nitrogen concentration in these films

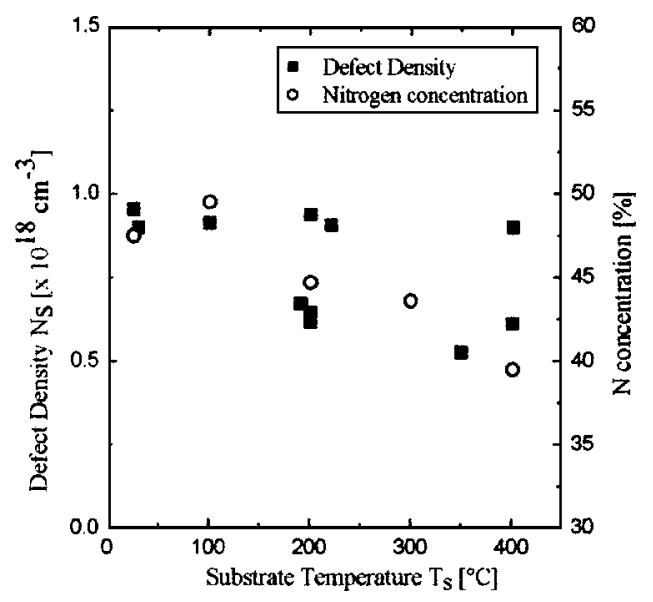

FIG. 2. Dependence of the defect density $N_{s}$ (obtained from ESR) and the corresponding nitrogen concentration (determined from EELS) for

a-CN $\mathrm{CN}_{x}$ :LL films, as a function of substrate temperature $T_{s}$ a function of substrate temperature $T_{s}$.
Downloaded 30 Mar 2009 to 131.227 .178 .132 . Redistribution subject to AIP license or copyright; see htt

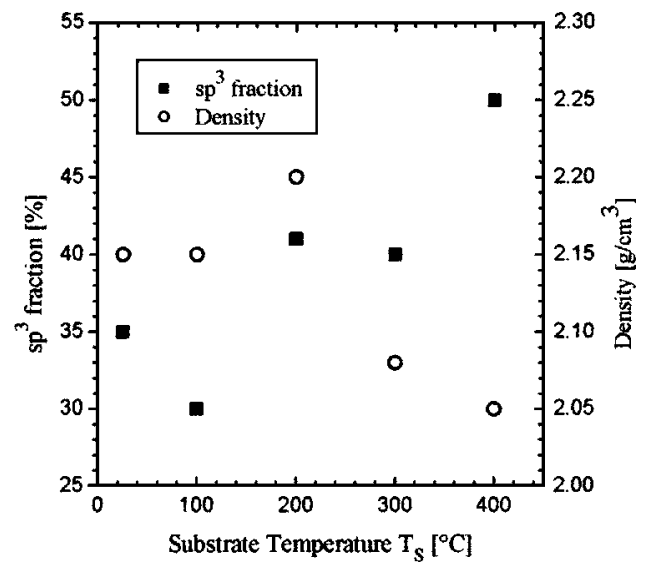

FIG. 3. Dependence of the $\mathrm{sp}^{3}$ fraction and density of valence electrons $\rho$ for a-CN $\mathrm{CN}_{x}$ :LL films, as a function of substrate temperature $T_{s}$, obtained by electron-energy-loss spectroscopy.

prepared layer-by-layer is almost constant as a function of the substrate temperature, showing the direct link between the defect density and the nitrogen concentration.

Figure 3 shows the dependence of the $s p^{3}$ fraction and density $\rho$ for the a-CN $\mathrm{CN}_{x}$ :LL films as a function of substrate temperature $T_{s}$ as obtained by EELS. The results suggest that the films have a unique composition, as they show an unusual increase in the $s p^{3}$-hybridized carbon fraction concomitant with a decrease in the density $\rho$ of free carriers. Because of the almost constant nitrogen concentration as a function of substrate temperature, we suggest that the increased substrate temperature affords higher atomic hydrogen mobility in the films, resulting in more $s p^{3}$ bonding, in the form of $\mathrm{C}-\mathrm{H}$ (and $\mathrm{C}-\mathrm{H}-\mathrm{N}$ ) states. Generally, with higher atomic mobility of $\mathrm{C}$ and $\mathrm{N}$ atoms, a more $s p^{2}$-rich structure is formed, with a predominance of the planar bonds. The proposed higher density of $\mathrm{C}-\mathrm{H} s p^{3}$ states results in a lower overall density of the films, as measured. Furthermore, $\mathrm{C}-\mathrm{N} s p^{3}$ bonds would be more stable than $\mathrm{C}=\mathrm{N} s p^{2}$ bonds at higher temperatures, consistent with the measured $s p^{3}$ fractions.

The dependence of the optical energy gap $E_{04}$ and Urbach energy $E_{U}$ obtained from the PDS for the a-CN $\mathrm{CN}_{x}$ :LL films as a function of substrate temperature $T_{s}$ is shown in Fig. 4. The $E_{04}$ was determined from the energy at which the absorption coefficient $\alpha$ equals $10^{4} \mathrm{~cm}^{-1}$. The $E_{U}$ describes the curvature of the band edge of the tail state. The $E_{04}$ and

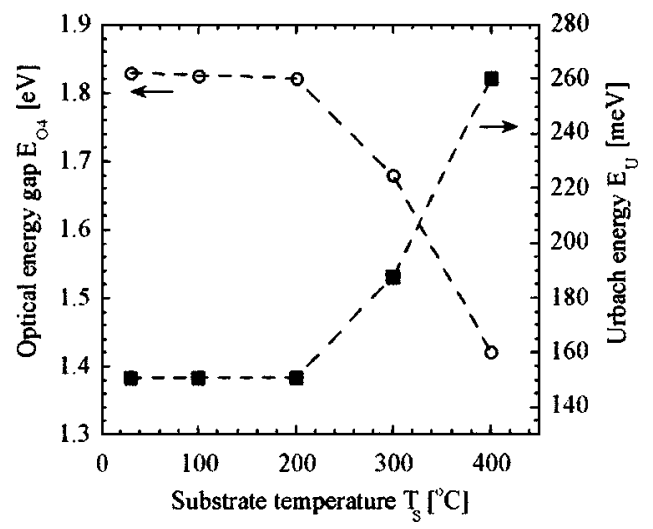

FIG. 4. Dependence of the optical energy gap $E_{04}$ and Urbach energy $E_{U}$, obtained from photothermal deflection spectroscopy, for a-CN $\mathrm{CN}_{x}$ : LL films as

a function of substrate temperature $T_{s}$. 
$E_{U}$ are approximately constant until $200^{\circ} \mathrm{C}$ and then begin to converge and crossover at $350{ }^{\circ} \mathrm{C}$. The increase in $E_{U}$ is related to the amount of disorder near the band edge. This may be explained by the free $\mathrm{N}$ forming clusters of $\mathrm{C}-\mathrm{N} s p^{2}$ states leading to the lower defect and physical density, and also a decrease in the $E_{04}$ band gap. We believe this clustering to predominate the $\pi$ component of the valence-band edge, for in optical absorption, the filled states are excited to empty antibonding states. If the $\mathrm{C}-\mathrm{N} s p^{2}$ cluster is present, with or without lone pair states at the $\pi$ band, this would give a broader tail.

The data shown in Fig. 1 gives a very interesting insight into the properties of these films. It firstly gives the shape of the convoluted bonding and antibonding states in which the bonding side probably dominates. It also gives a very large dynamic range of values, indicative of the huge difference in mobility values between high-energy extended states and highly localized $\pi$ states. The $y$ axis of the figure, as it stands, is equivalent to the uncorrected "figure of merit" for a single-carrier photoconductor, and will be a combination of the carrier absorption, recombination time, and convoluted mobility as a function of energy. There is a case for a twoGaussian fit to the data shown, with the first peak attributed to $s p^{2}$ states based upon $\mathrm{C}, \mathrm{C}-\mathrm{N}$, and some lone pair bonds. The higher energy peak at $6 \mathrm{eV}$ has been observed by many experiments, including the EELS low-loss region, and is attributed to the extended state conduction, or the highmobility region. If we are now to consider the data below $4.7 \mathrm{eV}$ for which we have accurate absorption data $(\alpha)$, based on optical measurements, it is possible to obtain the corrected $\mu \tau$ product. This gives a change in the photon number in the range of $10^{-11}-10^{-8} \mathrm{~cm}^{2} \mathrm{~V}^{-1}$, for the energy of 3.5-4.7 eV, with the $y$ axis in Fig. 1 transported by eight orders of magnitude to lower values. These absolute values are significant, for if we assume the recombination time to be in the order of $10^{-9}-10^{-8} \mathrm{~s}^{13}$ the resulting lower bound mobility is in the order of $10^{-3}-10^{-2} \mathrm{~cm}^{2} \mathrm{~V}^{-1} \mathrm{~s}^{-1}$ for the carriers (electrons) at an energy of $3.5 \mathrm{eV}$, increasing to around $1-10 \mathrm{~cm}^{2} \mathrm{~V}^{-1} \mathrm{~s}^{-1}$ for the $5 \mathrm{eV}$ states. These values are further justified as the mobility, close to the Fermi level, has been measured to be around $10^{-9}-10^{-6} \mathrm{~m}^{2} \mathrm{~V}^{-1} \mathrm{~s}^{-1}$. ${ }^{14,15}$ Higher values of $1-10 \mathrm{~cm}^{2} \mathrm{~V}^{-1} \mathrm{~s}^{-1}$ have also been observed under special conditions of nanostructuring in the a-CN $\mathrm{CN}_{x}$ films ${ }^{16}$ and under superlattice conditions. ${ }^{17}$ The small variation in the data as a function of substrate temperature is expected with the evolution of the bonding, especially the $\mathrm{C}-\mathrm{N} s p^{2}$ lone pair states and band gap. The variation is small, and we believe this could be due to the little variation observed in the unpaired defects measured by ESR.

In conclusion, we report high photosensitivity values of $10^{5}$ at monochromatic light with photon energy of $6.2 \mathrm{eV}$, with an energy flux of $5 \mu \mathrm{W} / \mathrm{cm}^{2}$. We show the movement of the resultant photoconductive spectrum as a function of substrate growth temperature, and postulate the existence of a $\mathrm{C}-\mathrm{N} s p^{2}$ lone pair state that dominates the recombination process close to the $\pi$ bonding states. The layer-by-layer growth process gives quite unexpected results in terms of the microstructure of the film with high $s p^{3}$ bonding and low band gap at high temperatures. This is explained by invoking the restructuring process that takes place during the hydrogen plasma treatment step, through the formation of $s p^{3}$ bonds with the introduction of hydrogen at higher substrate temperatures. Unlike previous studies, we do not see a significant dependence of the photoconductivity on the unpaired defect spins measured by ESR. The data shows this novel synthesis route of layer-by-layer growth of a-CN $\mathrm{CN}_{x}$ :LL by nitrogen radical sputtering to be a candidate for photodetector materials as well as photoconductors.

This research was supported partly by the Grant-in-Aid to Gifu University from the Ministry of Education, Sports, and Culture, Science and Technology, Japan. One of the authors (S.R.P.S) is thankful for a Monbusho-Gakusin Advanced Fellowship, Ministry of Education, Japan, which allowed this work to be conducted at Gifu.

${ }^{1}$ A. Ilie, O. Harel, N. M. J. Conway, J. Robertson, and W. I. Milne, J. Appl. Phys. 87, 789 (2000).

${ }^{2}$ G. A. J. Amaratunga, V. S. Veerasamy, W. I. Milne, C. A. Davis, S. R. P. Silva, and H. S. MacKenzie, Appl. Phys. Lett. 63, 370 (1993).

${ }^{3}$ N. M. J. Conway, A. Ilie, J. Robertson, W. I. Milne, and A. Tagliaferro, Appl. Phys. Lett. 73, 2456 (1998).

${ }^{4}$ N. Takada, K. Arai, S. Nitta, and S. Nonomura, Appl. Surf. Sci. 113-114, 274 (1997).

${ }^{5}$ E. Briotman, N. Hellgran, K. Järrendahl, M. P. Johansson, S. Olafsson, G. Radnóczi, J.-E. Sundgren, and L. Hultman, J. Appl. Phys. 89, 118 (2001).

${ }^{6}$ S. R. P. Silva, J. Robertson, Rusli, G. A. J. Amaratunga, and J. Schwan, Philos. Mag. B 74, 369 (1996).

${ }^{7}$ M. Zhang, Y. Nakayama, and M. Kume, Solid State Commun. 110, 679 (1999).

${ }^{8}$ T. Heitz, C. Godet, J. E. Bouree, B. Drevillon, and J. P. Conde, Phys. Rev. B 60, 6045 (1999).

${ }^{9}$ A. Grill, V. Patel, and C. Jahnes, J. Electrochem. Soc. 145, 1649 (1998); M. Aono, S. Nitta, T. Katsuno, T. Itoh, and S. Nonomura, Appl. Surf. Sci. 159-160, 341 (2000).

${ }^{10}$ S. Nitta and T. Katsuno, in Amorphous Carbon, edited by S. R. P. Silva (IEE, United Kingdom, 2003), pp. 336-338.

${ }^{11}$ S. Souto, M. Pickholz, M. C. dos Santos, and F. Alvarez, Phys. Rev. B 57, 2536 (1998).

${ }^{12}$ F. Alvarez, M. C. dos Santos, and P. Hammer, Appl. Phys. Lett. 73, 3521 (1998).

${ }^{13}$ Y. Masumoto, H. Kunitomo, S. Shionoya, H. Menekata, and H. Kukimoto, Solid State Commun. 51, 209 (1984).

${ }^{14}$ F. J. Clough, W. I. Milne, B. Kleinsorge, J. Robertson, G. A. J. Amaratunga, and B. N. Roy, Electron. Lett. 32, 319 (1996).

${ }^{15}$ A. Foulani, C. Laurent, B. Mebarki, and Y. Segui, J. Appl. Phys. 80, 470 (1996).

${ }^{16}$ J. Schwan, V. Batori, S. Ulrich, H. Erhardt, and S. R. P. Silva, J. Appl. Phys. 84, 2071 (1998).

${ }^{17}$ S. R. P. Silva, G. A. J. Amartunga, C. N. Woodburn, M. E. Welland, and S. Haq, Jpn. J. Appl. Phys., Part 1 33, 6458 (1994). 